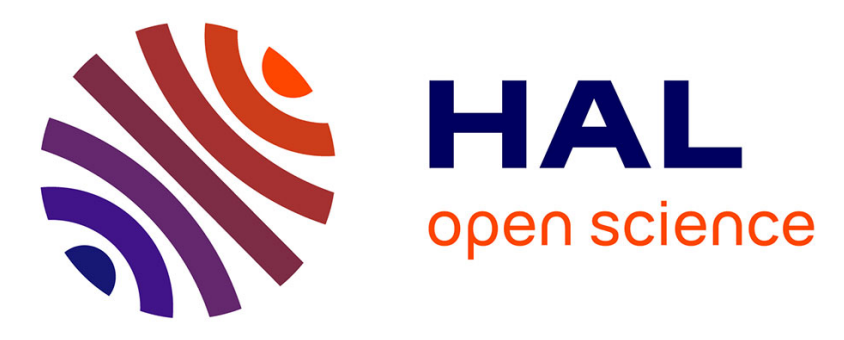

\title{
Ingot wavefront sensor: from the Fourier End2End numerical simulation to the LOOPS test bench
}

Carmelo Arcidiacono, Simone Di Filippo, Davide Greggio, Kalyan Kumar

Radhakrishnan Santakumari, Elisa Portaluri, Maria Bergomi, Valentina

Viotto, Demetrio Magrin, Roberto Ragazzoni, Luca Marafatto, et al.

\section{To cite this version:}

Carmelo Arcidiacono, Simone Di Filippo, Davide Greggio, Kalyan Kumar Radhakrishnan Santakumari, Elisa Portaluri, et al.. Ingot wavefront sensor: from the Fourier End2End numerical simulation to the LOOPS test bench. SPIE Astronomical Telescopes + Instrumentation,, Dec 2020, Online Only, United States. pp.250, 10.1117/12.2562355 . hal-03225140

\section{HAL Id: hal-03225140 \\ https://hal.science/hal-03225140}

Submitted on 8 Jun 2021

HAL is a multi-disciplinary open access archive for the deposit and dissemination of scientific research documents, whether they are published or not. The documents may come from teaching and research institutions in France or abroad, or from public or private research centers.
L'archive ouverte pluridisciplinaire $\mathbf{H A L}$, est destinée au dépôt et à la diffusion de documents scientifiques de niveau recherche, publiés ou non, émanant des établissements d'enseignement et de recherche français ou étrangers, des laboratoires publics ou privés. 


\title{
Ingot Wavefront Sensor: from the Fourier End2End numerical simulation to the LOOPS test bench
}

\author{
Carmelo Arcidiacono ${ }^{\mathrm{a}, \mathrm{c}}$, Simone Di Filippo ${ }^{\mathrm{a}, \mathrm{b}, \mathrm{c}}$, Davide Greggio ${ }^{\mathrm{a}, \mathrm{c}}$, Kalyan Kumar \\ Radhakrishnan Santhakumari ${ }^{\mathrm{a}, \mathrm{c}}$, Elisa Portalurid ${ }^{\mathrm{d}, \mathrm{c}}$, Maria Bergomi $^{\mathrm{a}, \mathrm{c}}$, Valentina Viotto ${ }^{\mathrm{a}, \mathrm{c}}$, \\ Demetrio Magrin $^{\mathrm{a}, \mathrm{c}}$, Roberto Ragazzoni ${ }^{\mathrm{a}, \mathrm{b}, \mathrm{c}}$, Luca Marafatto ${ }^{\mathrm{a}, \mathrm{c}}$, Marco Dima ${ }^{\mathrm{a}, \mathrm{c}}$, Jacopo \\ Farinato $^{\mathrm{a}, \mathrm{c}}$, Pierre Janin-Potiron ${ }^{\mathrm{e}, \mathrm{f}}$, Thierry Fusco ${ }^{\mathrm{e}, \mathrm{f}}$, Benoit Neichel ${ }^{\mathrm{e}}$, Olivier Fauvarque ${ }^{\mathrm{e}}$, and \\ Lauren Schatz \\ anAF - Osservatorio Astronomico di Padova, Vicolo dell'Osservatorio 5, I-35122,Padova, Italy \\ ${ }^{\mathrm{b}}$ Dipartimento di Fisica e Astronomia, Università degli Studi di Padova, Vicolo \\ dell'Osservatorio 3, I-35122 Padova, Italy \\ ${ }^{c}$ ADONI, Laboratorio Nazionale di Ottica Adattiva, Italy

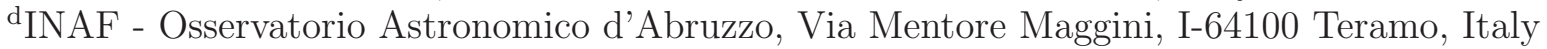 \\ eAix Marseille Univ, CNRS, CNES, LAM, Marseille, France \\ ${ }^{f}$ ONERA The French Aerospace Laboratory, F-92322 Châtillon, France \\ ${ }^{\text {g} T h e ~ U n i v e r s i t y ~ o f ~ A r i z o n a, ~ C o l l e g e ~ o f ~ O p t i c a l ~ S c i e n c e s, ~} 1630$ E University Blvd, Tucson, AZ \\ 85719, USA
}

\begin{abstract}
The Sodium Laser Guide Star (LGS) is an elongated object in a 3D volume. This produces a significant elongation on many of the Shack-Hartmann wavefront sensor spots that the ELT instruments use for wavefront sensing. The Ingot Wavefront Sensor (I-WFS) has been proposed as a possible solution to deal with the 3D nature of the LGS. We developed an end-to-end numerical tool of the I-WFS to perform system analysis of a closed-loop complete system. We considered the generation of the input turbulence, deformable mirror definition and control, and, of course details, of the I-WFS. It needs a 3-Dimensional description to fully take into account the elongation effect across the vertical (propagation) axis. The I-WFS has been simulated similarly to the Pyramid Wavefront Sensor, as a combination of Foucault knife-edge sensors. In parallel to numerical simulations development, we had the opportunity to perform laboratory testing of the I-WFS at the LOOPS Adaptive Optics test bench (at LAM), using a Spatial Light Modulator. This device is able to produce a high definition phase mask that can mimic two-dimensional I-WFS behaviour. In this framework, we report a preliminary discussion both for the simulations and the closed-loop data analysis.
\end{abstract}

Keywords: Adaptive Optics, Wavefront Sensing, Ingot WFS, Numerical Simulations, LGS, Sodium Laser Guide Star, ELT

\section{INTRODUCTION}

The Sodium Laser Guide Star ${ }^{1}$ (LGS) device is one of the key components of any advanced telescope providing adaptive optics ${ }^{2-5}$ instruments. LGSs give to the telescope the capability to enlarge the sky coverage creating an artificial reference star, to compensate for the absence of a sufficiently bright star for wavefront sensing. On the other hand, LGSs are not point-like sources, but elongated objects in the three-dimensional space. This is due to the intrinsic nature of the Sodium layer, which is located at about $90 \mathrm{~km}$ in the atmosphere and extended for approximately $20-25 \mathrm{~km} .{ }^{6}$ This layer, not only has a specific thickness, but also a particular vertical density distribution, both varying spatially and temporally. ${ }^{7}$ The Ingot Wavefront Sensor (I-WFS), ${ }^{8}$ has been

Further author information: (Send correspondence to C.A.)

C.A.: E-mail: carmelo.arcidiacono@inaf.it, Telephone: +39049 8293414

S.D.F.: E-mail: simone.difilippo@inaf.it, Telephone: +39049829 1234 
designed to cope with this typical elongation of the LGS and presented as novel pupil-plane wavefront sensor in the framework of the Extremely Large Telescope ${ }^{9}$ (ELT) project. The LGS produces a significant elongation on many of the Shack-Hartmann wavefront sensor (SH-WFS) spots, especially for large telescopes. The effect is even more significant for the ELT instruments that use SH-WFSs for the wavefront sensing and benefit of LGSs fired from launching telescopes placed at the side of the primary mirror.

We developed an end-to-end numerical tool to perform analysis of a closed-loop system. We considered the generation of the input turbulence, deformable mirror definition and control, and, of course, details of the I-WFS. To fully take into account the elongation effect across the vertical (propagation) axis the I-WFS has to consider a three-dimensional description. The wavefront propagation in the three-dimensional volume requires some ray tracing. ${ }^{10}$ Ray tracing is heavy in terms of required computing resources, and it is not convenient for control optimization studies. Such a solution would limit the kind of analysis we want to perform: using the numerical tool we aim to get insights about noise limit, optimal gain, best pupil geometry and performing slope calculation algorithm. Both in the numerical simulation and the laboratory framework, we considered a two-dimensional description of the I-WFS. It has been tested at the LAM-ONERA On-sky Pyramid Sensor LOOPS ${ }^{11}$ bench at Laboratoire d'Astrophysique de Marseille (LAM), in a quasi-real Adaptive Optics (AO) system using two Spatial Light Modulators (SLM) for the generation of the two-dimensional phase mask representing the I-WFS faces and, separately, the phase screen and the corrector (working as a deformable mirror). For more and further details about the I-WFS, we direct the reader to the original papers ${ }^{8,12,13}$ and further developments. ${ }^{14-18}$ In section 2 we describe the I-WFS, in section 4 the LOOPS bench setup, in section 3 we introduce the numerical tool used to produce the simulation presented in section 5 and discussed in section 6 .

\section{THE INGOT WAVEFRONT SENSOR}

The actual design of the I-WFS is based on an evolution of the four faces Pyramid Wavefront Sensor ${ }^{19}$ (P-WFS), based on a refractive prism. The I-WFS, on the contrary, is composed of a reflecting roof, which allows splitting the incoming LGS light into three beams, two reflected and the third is passing unperturbed towards the following obejective. These three separated beams are re-imaged in as many pupils at the conjugated plane, as shown in Figure 1. The I-WFS has a reflective roof with its edge positioned along the elongation axis of the LGS image.

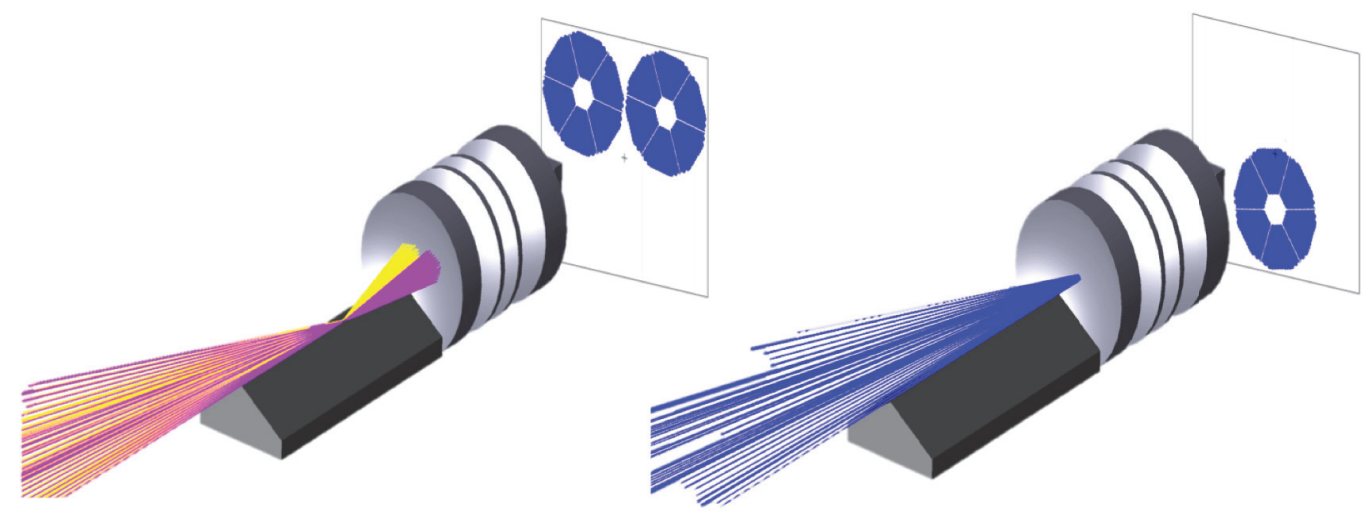

Figure 1. Conceptual design of the 3-faces I-WFS

The roof is placed on the LGS image such that it can intercept only a portion of the LGS (left side of Figure 1), while the remaining portion of the LGS does not intercept the prism and produces the third pupil (right side of Figure 1). One can linearly combine the intensity map on the three pupils to calculate signals proportional to the first derivative of the incoming wavefront, like for the P-WFS. The signals are defined using the following 
relations:

$$
\begin{aligned}
& S_{x}=\frac{B-C}{A+B+C}-\frac{B_{r e f}-C_{r e f}}{A_{r e f}+B_{r e f}+C_{r e f}}, \\
& S_{y}=\frac{A}{A+B+C}-\frac{A_{r e f}}{A_{r e f}+B_{r e f}+C_{r e f}} .
\end{aligned}
$$

Where $x$ and $y$ describe respectively the movements orthogonal and along the elongation direction. $A$ is the intensity on the transmitted pupil, and $B$ and $C$ are the intensities on the two reflected pupils.

\section{SIMULATION TOOL DESCRIPTION}

In the simulations we modelled the I-WFS similarly to the P-WFS, ${ }^{20}$ as a combination of Foucault knife-edge sensors. We simplified the computation through a far-field configuration involving only a sequence of Fourier transforms. The complex electromagnetic field on the I-WFS edges is computed as the Fourier transform of the phase in the aperture. We represent the ingot faces as a phase-mask that splits (tilting) the light. The image of the I-WFS focal plane (pupil images) is simulated through the Fourier transform of the complex array in output from the previous step. The P-WFS is typically modulated; ${ }^{19}$ however here we do not consider modulation because of the computational workload and especially because a similar algorithm is already exploited to generate the extension of the LGS source. The two effects, modulation and extension, of the reference source, are almost equivalent: the extended source can be modelled as an elaborated form of modulation. We approximate the elongated spot to be two-dimensional, as it was also simulated on the LOOPS bench, and divided it into pointlike sources, to cover the angular extension observed from the ground.

We adapted the already available structure of the MAO code ${ }^{21,22}$ to build the algorithm for the closed-loop system. The module is approximated into its main components: input turbulence, WFS, deformable mirror, and control loop. For the input turbulence, we foresee two options: the turbulence is injected using the deformable mirror, or the phase is generated by an external input (e.g. moving phase screen or spatial light modulator). In the numerical simulation, the phase is ultimately a moving random phase screen or a combination of mirror

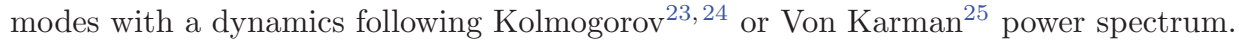

The deformable mirror can be computed as a linear combination of the vectors composing a Zernike or actuators influence function modal base. The control loop is a pure integrator with user-defined frequency expressed in frame delay and bandwidth modulated by a gain value. The comparison of the laboratory results with the outcomes of the simulation analysis provides a better insight into the ingot behaviour in the closed-loop.

\section{THE INGOT WAVEFRONT SENSOR AT THE LOOPS BENCH}

The LOOPS ${ }^{11}$ is an adaptive optics facility hosted at LAM and focusing on the study of Fourier-filtering wavefront sensors. The LOOPS bench allows testing the I-WFS in a quasi-real AO closed-loop context. The optical design of the LOOPS test bench is shown in Figure 2:

The bench is divided into four blocks: (I) a common path, (II) a metrology path, (III) a classic PWFS path, and (IV) a Fourier-based WFS path, as described in previous work. ${ }^{26}$ The peculiarity of this facility is the presence of a SLM, shown in block (IV). This device is a Hamamatsu SLM (LCOS-SLM X13138) with $1024 \times 1280$ pixels (with a $12.5 \mu \mathrm{m}$ pitch) that can produce arbitrary phase masks modifying its shape. It uses liquid crystal technology and exploits the birefringence properties of the crystals to produce configurable phase masks. It is located in the focal plane to reproduce a two-dimensional phase mask of the wavefront sensor. Other components of this facility, used to test the I-WFS are described in the following:

- Monochromatic light source (S in Figure 2) generated from a laser diode and feeding an optical fiber at the wavelength of $\lambda=660 \mathrm{~nm}$

- PI piezoelectric tip-tilt mirror (TTM in Figure 2). It produces the modulation of the point-like source. It is controlled in an open-loop mode and operated at $500 \mathrm{~Hz}$. 


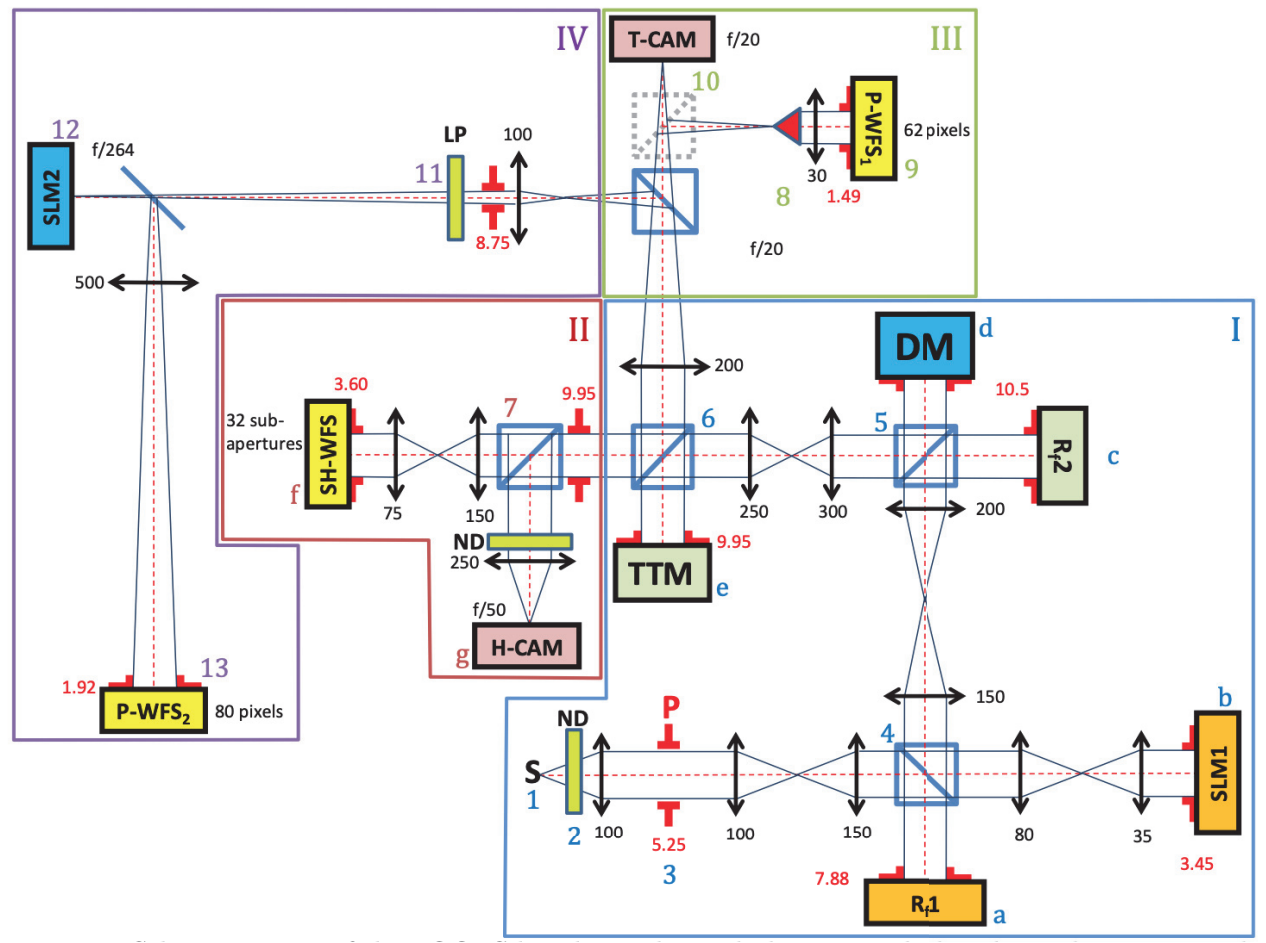

Figure 2. Optical Design of the upgraded LOOPS bench facility. (I) is the common path optics, (II) a metrology path, (III) the classic P-WFS path, and (IV) the Fourier-based WFS path including a Spatial Light Modulator (SLM).

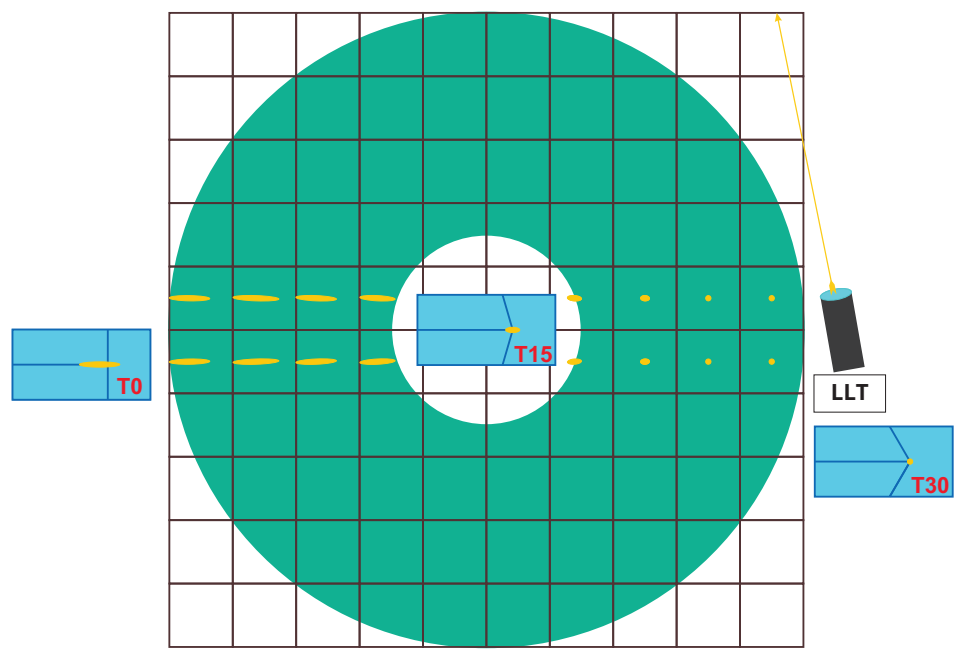

Figure 3. Picture shows some of the sub-aperture of a Shack-Hartmann like WFS as illuminated by a laser, fired on the side of the primary (from the Laser Launching Telescope (LLT)). Each sub-aperture has its own perspective of the emitting sodium profile. A different phase mask (here T0, T15 and T30) corresponds to the ingot figure projection as seen from the sub-aperture. The dimensions of masks, LLT and elongation are not in scale.

The I-WFS collapses different pupil viewpoints in one single device, see Figure 3. As seen from one single subaperture, the inclination of the I-WFS prism changes with the distance from the edge. The approximation of the three-dimensional geometry, cannot be reproduced in a two-dimensional system in a straightforward way. We opted for the realization in the model of three different configurations (perspectives), corresponding to three 
different shapes of the wavefront sensor phase masks. To realize the LGS reference on the bench, we used a fast tip-tilt mirror (TTM) to simulate the extension of the source by introducing an elliptical modulation to the available point source. We calculated the length of the extended source considering the ELT scenario and system parameters and $\approx 20 \mathrm{~km}$ sodium layer thickness: different portions of the pupil have correspondingly different geometry. We take into account three cases corresponding to different sub-apertures over the telescope pupil, starting from the closest to the laser launcher position (considering a single laser launcher located aside from the telescope), and ending on the one on the opposite edge of the pupil, considering, in addition, one in the middle. In such configuration, we found three different phase masks, which differ for their projection of the prism roof. These differences correspond in the two-dimensional picture to as many shapes, that we draw starting from the angle shown in Figure 4. In the following, we refer to these respectively as: T30, T15, and T0. On the bench we coupled these sub-apertures to as many different aspect ratios of the spot elongation in these cases of $1: 1$, 1:7 and 1:15. However, on the ELT the aspect ratio will be larger, respectively 1:1, 1:15 and 1:30: the longest elongation was not feasible using the tip-tilt mirror modulation.
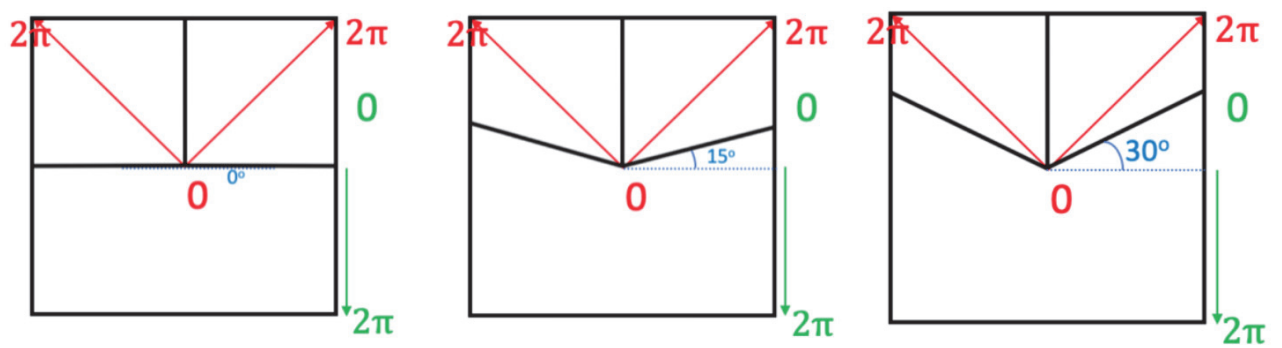

Figure 4. Ingot phase masks used to reproduce the three different and extreme cases used in the test. Left: ingot seen from the farther sub-aperture with respect to the laser launcher, (T0). Center: ingot seen from a middle positioned sub-aperture, (T15). Right: ingot seen from the closest sub-aperture to the laser launcher, (T30).

In this paper we report only about the test performed using a source aspect ratio of 1:7. Other elongations, but using as turbulence input device a deformable mirror instead of the SLM, have been already tested, and the results are available in a previous work: ${ }^{17}$ in 2019 , the LOOPS bench facility has been upgraded with the SLM. ${ }^{27}$ This new device is able to serve at the same time as a high-resolution deformable mirror and a turbulent phase screen generator, with a much higher resolution with respect to the previous configuration ${ }^{17}$ using the deformable mirror. The spatial resolution of the input phase increased from the 65 Zernike modes to the 230 Karhunen-Loéve modes used for our test. The latest optical design of the bench is the one shown in Figure 2.

In this new framework, we started testing the I-WFS for several combinations of source aspect ratios and wavefront sensor phase masks: here we report about test using the 1:7 aspect ratio and the T15 mask. We also performed the comparison with the P-WFS case by making the SLM produce a four-faces phase mask. In this work the SLM on a intermediate pupil plane applied a disturbance with a resolution of 320 pixels in the pupil diameter. Figure 5 shows, for the same frame, on the left the shape of the input turbulence, and on the right the complementary correction applied by the SLM.

Here, we report the characteristics of the input turbulence that has been created using the OOMAO ${ }^{28} \operatorname{code}$

- $r_{0}=0.9 m$

- Equivalent Telescope Diameter $=8 \mathrm{~m}$

- Wind speed $=10 \mathrm{~m} / \mathrm{s}$

- Bandwidth $=200 \mathrm{~Hz}$

- Seeing $=0.15 "$

- Calibration and correction made with $230 \mathrm{KL}$ modes 

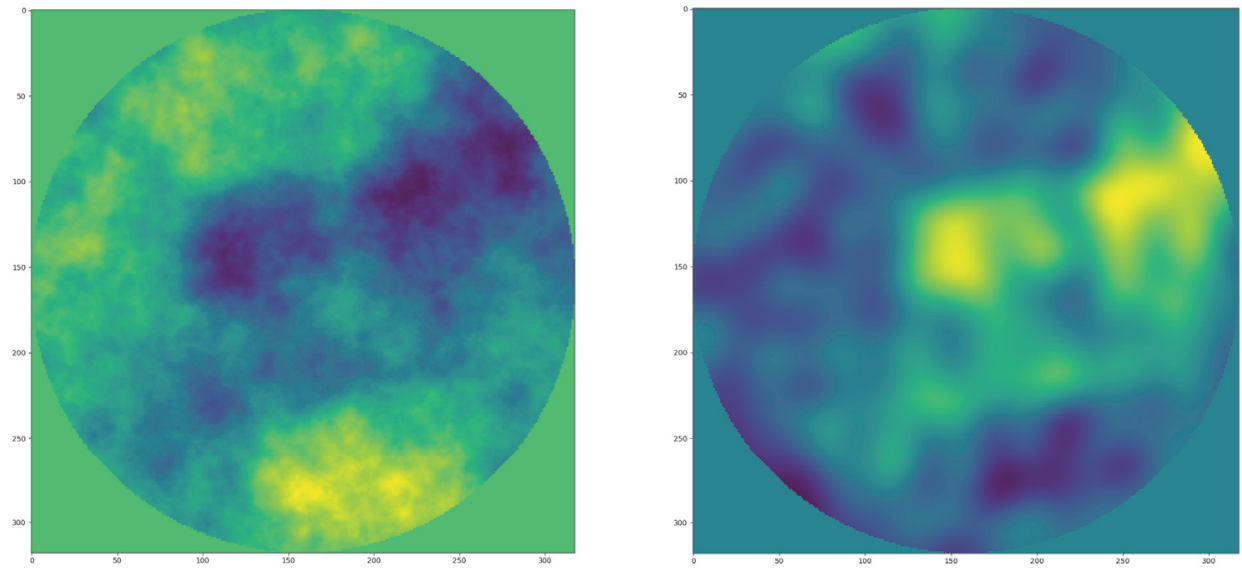

Figure 5. The two pictures freeze an instant of the loop evolution. On the left the phase map of the turbulence in input as processed by the SLM. On the right the complementary correction applied by the SLM as a phase map.

- Pupil dimension $=318 \times 318 \mathrm{px}$

- Turbulence history, length $=1000$ iterations

Note that, to make the calibration and calculate the interaction matrix, we used the signals relations shown in formula eq. 1. According to the input parameters, we report in this work the results for the configuration of the T15 phase mask for the 1:7 source aspect ratio for three cases and the P-WFS:

case $112.5 \%$ of the total flux (1/8th) in the transmitted pupil, using the signals calculated following eq. 1

case $212.5 \%$ of the total flux (1/8th) in the transmitted pupil, using the fluxes measured from the pupils

case $325 \%$ of the total flux (2/8th) in the transmitted pupil, using the signals calculated following eq. 1

case 4 P-WFS using the fluxes measured from the pupils

Figure (6) shows the Standard Deviation of the Wavefront Error map during the iterations for open and closed loop for the cases 1, 3, and 4. As the plot in Figure 6 shows, both I-WFS and P-WFS well perform in the closedloop scenario. Apparently the two I-WFS curves superimpose on the open-loop line more than the P-WFS does. This effect is due to some delay in the activation of the loop. While if we look and the residual's mean values of the three cases, we can easily note that the I-WFS shows a lower order of residual aberrations (i.e., a lower mean value), even if the different is negligible.

Besides, we report a comparison between "case 1" and "case 2". For both of them, the flux in the transmitted pupil is equal to the $12.5 \%$ of the total, as shown in Figure 7 Here, we can notice from the plot that "case 2" shows a residual mean value lower than "case 1". This very preliminary result could suggest to us to consider as alternative to the eq. 1, the possibility to use as signals the normalized fluxes of the three pupils. We need to take into account, that this is a single result, that needs to be provided with more statistics.

\section{DETAILING THE SIMULATED CASE}

We performed several simulations using in input the phase map used also as input for the SLM on the LOOPS bench. The modal base used for the modelling of the deformable mirror in the SLM, was not exported into file. We substituted in the numerical simulations the Karhunen-Loéve modal base used in LOOPS, with a 200 Zernike polynomials modal base, defined on a full circular pupil. The pupil amplitude map and the complex phase array were zero-padded in order to get a resolution on the I-WFS roof of $4 \lambda / D$ per pixel $(\lambda=$ wavelength, $D$ is telescope diameter). 


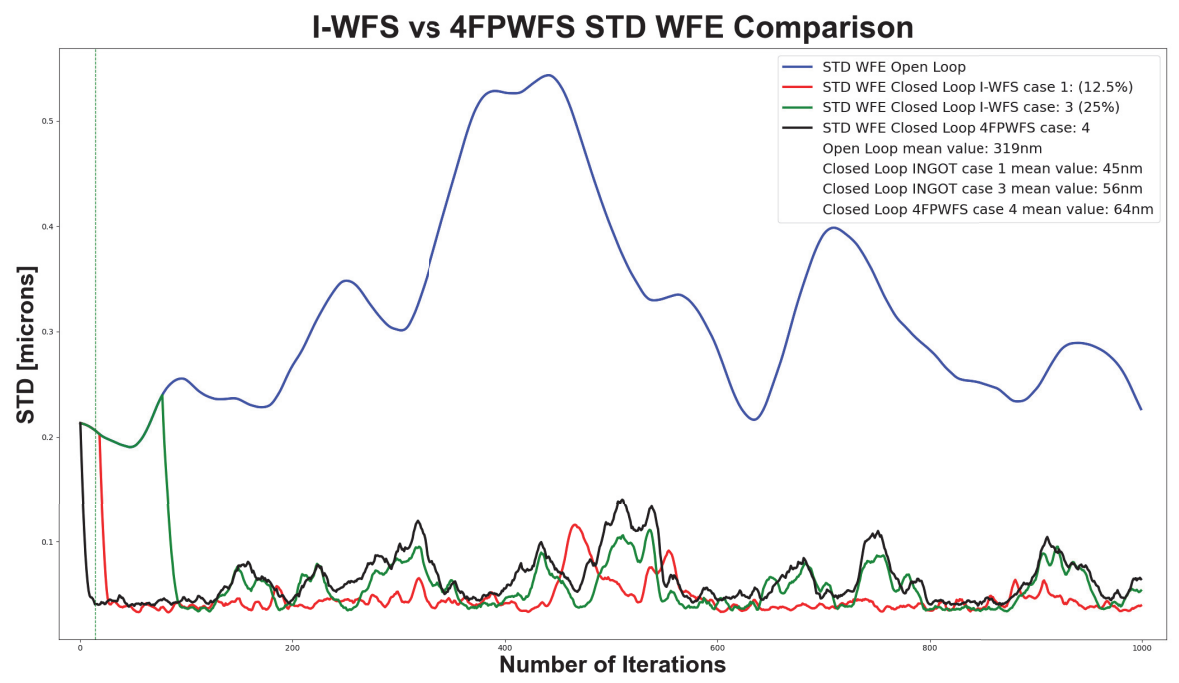

Figure 6. Standard Deviation Wavefront Errors Open-Closed loop comparison between I-WFS cases (1-3) and P-WFS.

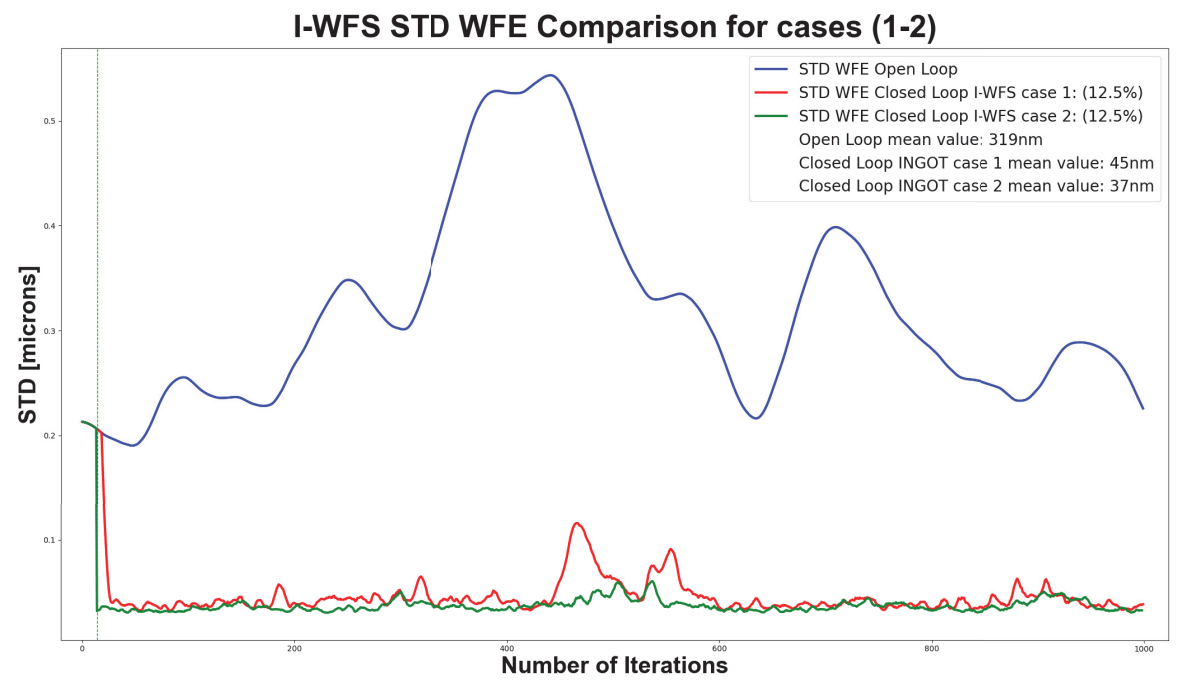

Figure 7. Standard Deviation Wavefront Errors Open-Closed loop comparison between cases (1-2).

In this test, we did not include nor photon noise neither read noise of the detector.

We also decided to remove from the turbulent phase in input and from the modal base the tip and tilt terms. One simple reason is that LGS should not compensate for atmospheric tip-tilt. Moreover, we already noticed in previous simulations ${ }^{17}$ performed using smaller Field of View (FoV) (1") that the circular wrapping of the numerical FFT may cause instabilities: since we noticed suspicious instabilities spikes we preferred to eliminate this possible source of problem.

Another possible issue is related to light spread out by diffraction. In the numerical simulation, we didn't address it properly and some light from the two reflected pupils in the top part of the detector pollutes the measurement on the transmitted single one. In the forthcoming simulations we are appropriately correcting the pupil position 
to overcome the issue.

In Figure 8 we present the results in terms of wavefront error standard deviation as measured for each iteration of the loop. We provide at first the same configurations used in the laboratory setup "case 1", "case 2" and "case 3", and in Figure 9 similar configurations but using a different ratio of the light split between transmitted and reflected pupils. These simulations were not converging as expected, most probably because of the extra noise introduced by diffracted light on the refracted pupil.

In order to counterbalance this effect we decided to change the illumination ratio, forcing more light on the refracted pupil and reducing in this way the relative intensity of the diffracted light.

The cases shown in Figure 9 correspond to the following configurations:

case $1 \mathrm{~b} 50 \%$ of the total flux in the transmitted pupil, using the signals calculated following eq. 1.

case $2 \mathrm{~b} 50 \%$ of the total flux in the transmitted pupil, using the fluxes measured from the pupils.

case 5 a functional case using a point source, no modulation, $50 \%$ of the total flux in the transmitted pupil, using the fluxes measured from the pupils.

Table 1. Input parameters used for the numerical simulations.

\begin{tabular}{|l|l|l|l|l|}
\hline \hline Pupil Geometry & $D$ & wavelength & FFT resolution & FoV \\
\hline Full Circular & $8 \mathrm{~m}$ or $318 \mathrm{px}$ & $\lambda=660 \mathrm{~nm}$ & $4 \lambda / D$ & 5 arcsec \\
\hline \hline
\end{tabular}

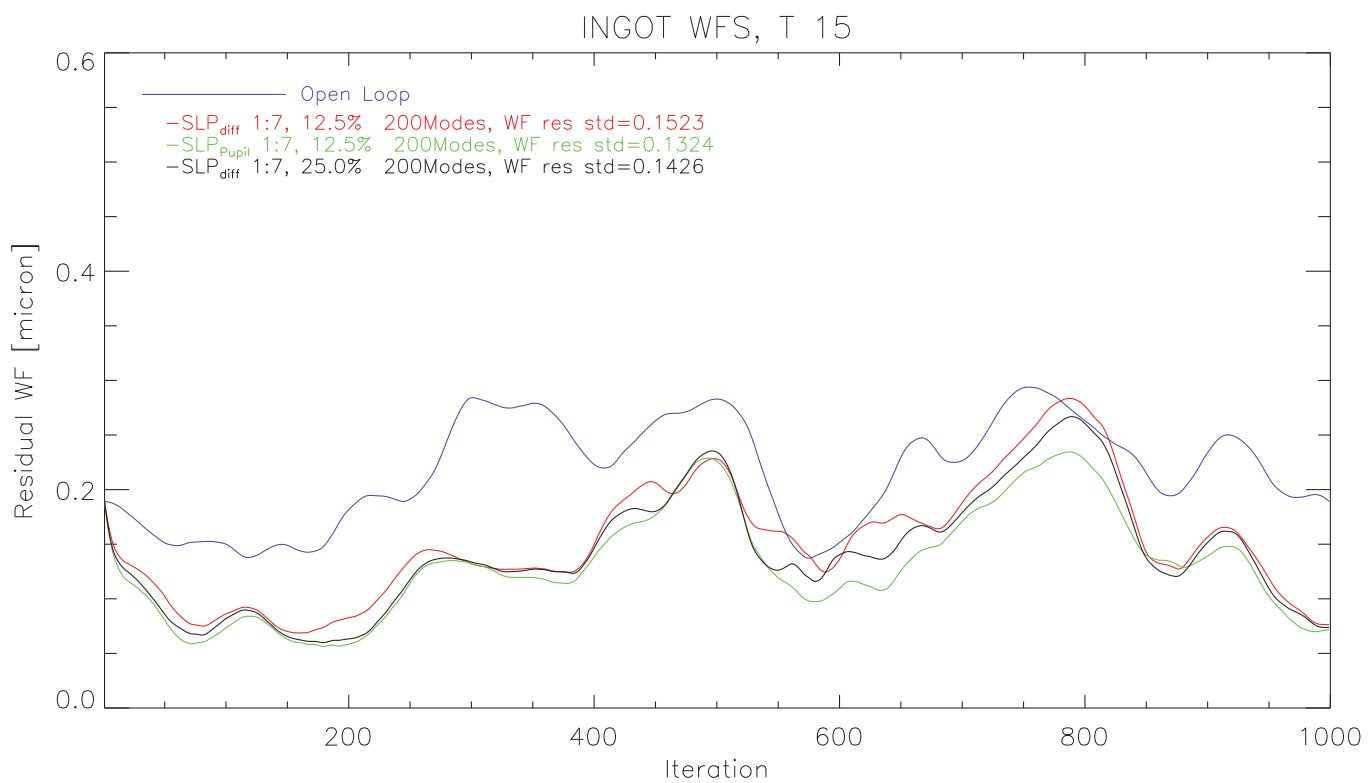

Figure 8. The plots show the closed loop residual phase standard deviation of the configurations corresponding to "case 1", "case 2" and "case 3" (in blue the open loop). For a better readability: in red using the slope computation of eq. 1 and 1:8 pupil illumination ratio, in green using normalized pupils instead than slopes, and finally in black the eq. 1 slopes and ratio $1: 4$

Comments on the results in the next section. 


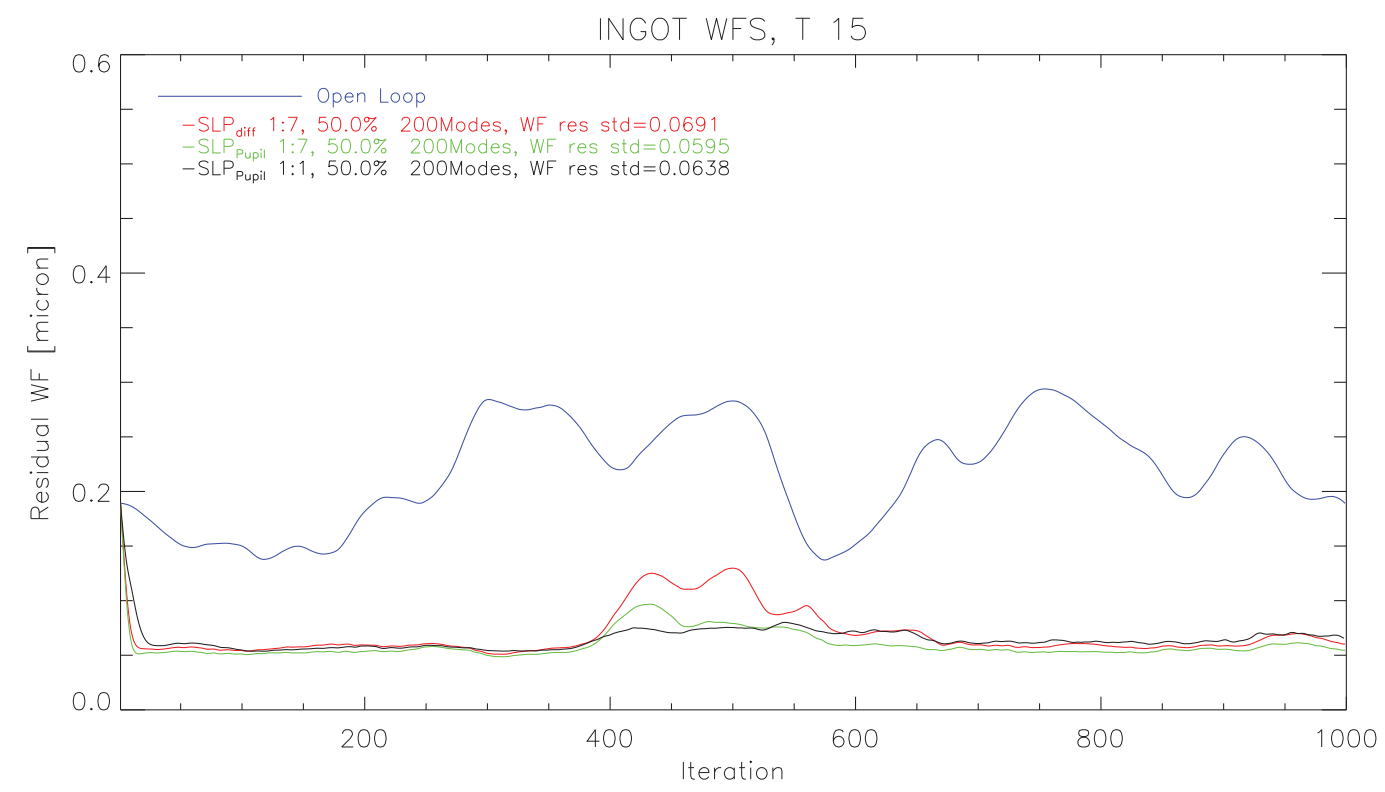

Figure 9. Referring to the "case 1b", "case 2b" and "case 5" described in Section 5. In short: in red the case with slope computation of eq. 1 , in green using normalized pupils, in black the functional test using a point-like source.

\section{TESTS AND SIMULATION COMPARISON}

LOOPS bench and numerical simulations provides insight about the behaviour of I-WFS in closed loop. A crucial point is the linearity of I-WFS response that we are addressing. Strictly related to this point is the optimal illumination of the refracted pupil versus the reflected two: both simulation and experiment show the need to close the loop with a non-zero reference slope vector. However, large amplitudes of the slope vector may introduce non-linearity issues (as the case of the P-WFS).

In terms of result the I-WFS performs slightly better of the P-WFS having an extended reference source. Or being less optimistic the performance is comparable. We need to explore different level of illumination and turbulence strength.

The numerical simulation provided similar values for the standard deviation of the residual phase to the LOOPS experiment (both in the range of 40-60nm). In the simulations we excluded the tip-tilt both from the input and the controlled modes: in the case of the laboratory we can assume that the contribute of the very low order modes is negligible since the input aberration is a pure atmospheric disturbance that shows a slowly evolving bent of the wavefront, and also vibration-free.

Theoretically, we expect using the parameters of the turbulence statistics, the number of modes used for correction and the bandwidth of the system (in section:4) a noise-free correction residual with standard deviation of $37 \mathrm{~nm}$ for the LOOPS setup and $39 \mathrm{~nm}$ for the simulation. Both values are in good agreement with the measured values (i.e., $45 \mathrm{~nm}$ and $60 \mathrm{~nm}$ ) that include also other error sources related to the bench realization and the IWFS sensitivity. For a cross-check, the values in open loop are respectively 650nm (assuming pure Kolmogorov turbulence) and $260 \mathrm{~nm}$ in the tip-tilt-free case: the turbulence statistic in open loop fits well with a turbulence spectrum with a finite outerscale.

The experiment and the simulations suggest to further investigate the optimal definition of the I-WFS measurement output formula, since the case using the normalized pupil intensities as vector for the multiplication for the control matrix in both cases provides marginally more stable results.

\section{CONCLUSION}

The Ingot Wavefront Sensor has been proposed as a novel pupil plane wavefront sensor, to cope with the intrinsic elongation effects of the Sodium Laser Guide Stars. In this work we review the status of both the numerical 


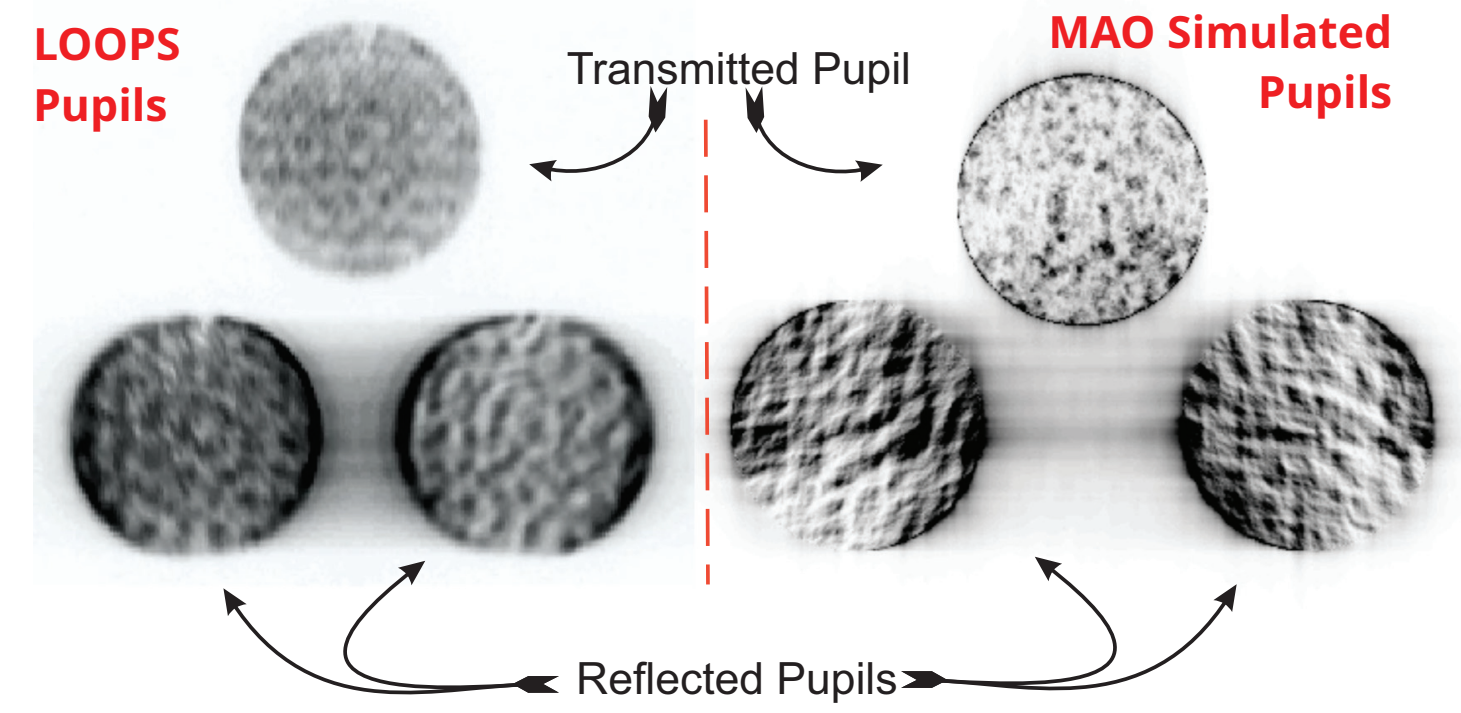

Figure 10. The figure shows the three pupil images arrangement for the laboratory and in the numerical simulations, respectively on the left and the right part of the image. $25 \%$ illumination vs $50 \%$ illumination of the transmitted pupil respectively.

simulation tool and the laboratory tests we are performing on the LOOPS bench at LAM about the I-WFS. In this configuration the LOOPS bench has been upgraded with a second SLM to increase the spatial resolution of the turbulent phase and the effect of high spatial frequencies both on the PSF and for the I-WFS measurements. We performed tests for a model of three different configurations (perspectives), corresponding to three shapes of the wavefront sensor phase masks, which differ from their projection of the prism roof. These phase masks have been identified considering three extreme cases: ingot seen from the farther sub-aperture of the telescope pupils with respect to the laser launcher. Ingot seen from a middle positioned sub-aperture, and ingot seen from the closest sub-aperture to the laser launcher. They have been called, respectively: T0, T15, T30. On the other hand, we coupled these sub-apertures to as many different aspect ratios of the source spot elongation, to reproduce the LGS real world extensions, for three cases: 1:1, 1:7, and 1:15. We reported, as example, the analysis made for the configuration 1:7 source aspect ratio and T15 phase mask. We showed which is the behaviour of the I-WFS in closed-loop, making in addition a comparison with the 4 Faces Pyramid Wavefront Sensor. We similarly updated the numerical simulation tool, derived from the MAO code developed for MAORY, to harmonize the model of the I-WFS to what was applied on the LOOPS bench. We performed numerical simulations matching the I-WFS geometry and mask configuration used on the trials on the LOOPS bench. The results are in good agreement, showing the ability of the I-WFS to close the adaptive loop using more than 200 modes on the corrector.

In the next months we foresee to solve the issues already identified (i.e., the diffracted light polluting the refracted pupil) and perform more extensive test about control strategies focusing on the achievable performance.

\section{ACKNOWLEDGMENTS}

This work is supported by the INAF Progetto Premiale "Ottica Adattiva Made in Italy per i grandi telescopi del futuro". The INAF team thanks the Laboratoire d'Astrophysique de Marseille (LAM) for their kind hospitality.

\section{REFERENCES}

[1] Thompson, L. and Gardner, C., "Artificial guide stars for adaptive imaging," Nature 330, 116 (Nov. 1987).

[2] Rabien, S., Ageorges, N., Barl, L., Beckmann, U., Blümchen, T., Bonaglia, M., Borelli, J. L., Brynnel, J., Busoni, L., Carbonaro, L., Davies, R., Deysenroth, M., Durney, O., Elberich, M., Esposito, S., Gasho, V., 
Gässler, W., Gemperlein, H., Genzel, R., Green, R., Haug, M., Hart, M. L., Hubbard, P., Kanneganti, S., Masciadri, E., Noenickx, J., Orban de Xivry, G., Peter, D., Quirrenbach, A., Rademacher, M., Rix, H. W., Salinari, P., Schwab, C., Storm, J., Strüder, L., Thiel, M., Weigelt, G., and Ziegleder, J., "ARGOS: the laser guide star system for the LBT," in [Adaptive Optics Systems II], Ellerbroek, B. L., Hart, M., Hubin, N., and Wizinowich, P. L., eds., Society of Photo-Optical Instrumentation Engineers (SPIE) Conference Series 7736, 77360E (July 2010).

[3] van Dam, M. A., Bouchez, A. H., Le Mignant, D., Johansson, E. M., Wizinowich, P. L., Campbell, R. D., Chin, J. C. Y., Hartman, S. K., Lafon, R. E., Stomski, Jr., P. J., and Summers, D. M., "The W. M. Keck Observatory Laser Guide Star Adaptive Optics System: Performance Characterization," Publications of the Astronomical Society of the Pacific 118, 310-318 (Feb. 2006).

[4] D’Orgeville, C., Diggs, S., Fesquet, V., Neichel, B., Rambold, W., Rigaut, F., Serio, A., Araya, C., Arriagada, G., Balladares, R., Bec, M., Boccas, M., Duran, C., Ebbers, A., Lopez, A., Marchant, C., Marin, E., Montes, V., Moreno, C., Petit Vega, E., Segura, C., Trancho, G., Trujillo, C., Urrutia, C., Veliz, P., and Vucina, T., "Gemini South multi-conjugate adaptive optics (GeMS) laser guide star facility on-sky performance results," in [Adaptive Optics Systems III], Ellerbroek, B. L., Marchetti, E., and Véran, J.-P., eds., Society of Photo-Optical Instrumentation Engineers (SPIE) Conference Series 8447, 84471Q (July 2012).

[5] Bonaccini Calia, D., Feng, Y., Hackenberg, W., Holzlöhner, R., Taylor, L., and Lewis, S., "Laser Development for Sodium Laser Guide Stars at ESO," The Messenger 139, 12-19 (Mar. 2010).

[6] Butler, D. J., Davies, R. I., Redfern, R. M., Ageorges, N., and Fews, H., "Measuring the absolute height and profile of the mesospheric sodium layer using a continuous wave laser," $A \& 4 A$ 403(2), 775-785 (2003).

[7] Pfrommer, T. and Hickson, P., "High resolution mesospheric sodium properties for adaptive optics applications," Astronomy \& Astrophysics 565, A102 (May 2014).

[8] Ragazzoni, R., Portaluri, E., Viotto, V., Dima, M., Bergomi, M., Biondi, F., Farinato, J., Carolo, E., Chinellato, S., Greggio, D., Gullieuszik, M., Magrin, D., Marafatto, L., and Vassallo, D., "Ingot Laser Guide Stars Wavefront Sensing," AO4ELT5 Proceedings (Aug. 2017).

[9] Gilmozzi, R. and Spyromilio, J., "The 42m European ELT: status," in [Society of Photo-Optical Instrumentation Engineers (SPIE) Conference Series], Proc. SPIE 7012 (2008).

[10] Portaluri, E., Viotto, V., Ragazzoni, R., Arcidiacono, C., Santhakumari, K. K. R., Bergomi, M., Greggio, D., Di Filippo, S., Farinato, J., and Magrin, D., "Evaluating the performance of an Ingot wavefront sensor for the ELT: good news from simulations," in [This conference], Proc. SPIE (2020).

[11] Janin-Potiron, P., Neichel, B., and Fusco, T., "Development of the adaptive optics testbed LOOPS for Fourier-based wavefront sensors demonstration and analysis," in [SF2A-2018: Proceedings of the Annual meeting of the French Society of Astronomy and Astrophysics], Di Matteo, P., Billebaud, F., Herpin, F., Lagarde, N., Marquette, J. B., Robin, A., and Venot, O., eds., Di (Dec. 2018).

[12] Ragazzoni, R., Greggio, D., Viotto, V., Di Filippo, S., Dima, M., Farinato, J., Bergomi, M., Portaluri, E., Magrin, D., Marafatto, L., Biondi, F., Carolo, E., Chinellato, S., Umbriaco, G., and Vassallo, D., "Extending the pyramid WFS to LGSs: the INGOT WFS," in [Adaptive Optics Systems VI], Close, L. M., Schreiber, L., and Schmidt, D., eds., Proc. SPIE 10703, 107033Y (July 2018).

[13] Viotto, V., Portaluri, E., Arcidiacono, C., Ragazzoni, R., Bergomi, M., Di Filippo, S., Dima, M., Farinato, J., Greggio, D., Magrin, D., and Marafatto, L., "Dealing with the cigar: preliminary performance estimation of an INGOT WFS," in [Adaptive Optics Systems VI], Close, L. M., Schreiber, L., and Schmidt, D., eds., Proc. SPIE 10703, 107030V (July 2018).

[14] Ragazzoni, R., Viotto, V., Portaluri, E., Bergomi, M., Greggio, D., Di Filippo, S., Radhakrishnan, K., Umbriaco, G., Dima, M., Magrin, D., Farinato, J., Marafatto, L., Arcidiacono, C., and Biondi, F., "Pupil plane wavefront sensing for extended and 3d sources," in [AO4ELT6], (2019).

[15] Viotto, V., Portaluri, E., Arcidiacono, C., Bergomi, M., Di Filippo, S., Greggio, D., Radhakrishnan, K., Dima, M., Farinato, J., Magrin, D., Marafatto, L., and Ragazzoni, R., "Ingot wavefront sensor: Simulation of pupil images," in [AO4ELT6], (2019).

[16] Portaluri, E., Viotto, V., Ragazzoni, R., Arcidiacono, C., Bergomi, M., Greggio, D., Radhakrishnan, K., Di Filippo, S., Marafatto, L., Dima, M., Biondi, F., Farinato, J., and Magrin, D., "Ingot wfs for lgss: First results from simulations," in [AO4ELT6], (2019). 
[17] Di Filippo, S., Greggio, D., Bergomi, M., Radhakrishnan, K., Portaluri, E., Viotto, V., Arcidiacono, C., Magrin, D., Marafatto, L., Dima, M., Ragazzoni, R., Janin-Portirond, P., Schatz, L., Neichel, B., Fauvarque, O., and Fusco, T., "Ingot wavefront sensor: from the optical design to a preliminary laboratory test," in [AO4ELT6], (2019).

[18] Santhakumari, K. K. R., Greggio, D., Di Filippo, S., Viotto, V., Portaluri, E., Arcidiacono, C., Dima, M., Luigi, L., Marafatto, L., Furierie, T., Bonora, S., and Ragazzoni, R., "Aligning and Testing the Ingot Wavefront Sensor in the Lab," in [This Conference], Proc. SPIE (2020).

[19] Ragazzoni, R., "Pupil plane wavefront sensing with an oscillating prism," Journal of Modern Optics 43 , 289-293 (1996).

[20] Vérinaud, C., "On the nature of the measurements provided by a pyramid wave-front sensor," Optics Communications 233(1-3), 27 - 38 (2004).

[21] Arcidiacono, C., Schreiber, L., Bregoli, G., Diolaiti, E., Foppiani, I., Cosentino, G., Lombini, M., Butler, R. C., and Ciliegi, P., "End to end numerical simulations of the MAORY multiconjugate adaptive optics system," in [Adaptive Optics Systems IV], Marchetti, E., Close, L. M., and Vran, J.-P., eds., Proc. SPIE 9148, 91486F (Aug. 2014).

[22] Arcidiacono, C., Schreiber, L., Bregoli, G., Foppiani, I., Oberti, S., Diolaiti, E., Agapito, G., Puglisi, A., Xompero, M., Cortecchia, F., Patti, M., Lombini, M., Busoni, L., Vérinaud, C., Felini, C., De Caprio, V., Cosentino, G., Ciliegi, P., Bellazzini, M., Feautrier, P., Esposito, S., and Ragazzoni, R., "Numerical simulations of MAORY MCAO module for the ELT," in [Adaptive Optics Systems VI], Close, L. M., Schreiber, L., and Schmidt, D., eds., Proc. SPIE 10703, 107034I (July 2018).

[23] Kolmogorov, A., "The Local Structure of Turbulence in Incompressible Viscous Fluid for Very Large Reynolds' Numbers," Akademiia Nauk SSSR Doklady 30, 301-305 (1941).

[24] Kolmogorov, A. N., "Dissipation of Energy in Locally Isotropic Turbulence," Akademiia Nauk SSSR Doklady 32, 16 (1941).

[25] Winker, D. M., "Effect of a finite outer scale on the zernike decomposition of atmospheric optical turbulence," J. Opt. Soc. Am. A 8(10), 1568-1573 (1991).

[26] Janin-Potiron, P., Chambouleyron, V., Schatz, L., Fauvarque, O., Bond, C. Z., Abautret, Y., Muslimov, E. R., Hadi, K. E., Sauvage, J.-F., Dohlen, K., Neichel, B., Correia, C. M., and Fusco, T., "Adaptive optics with programmable Fourier-based wavefront sensors: a spatial light modulator approach to the LAM/ONERA on-sky pyramid sensor testbed," Journal of Astronomical Telescopes, Instruments, and Systems 5(3), 1 - 10 (2019).

[27] Janin-Potiron, P., "Seeking for the ultimate wavefront sensor - Probing the free-parameters space of Fourierbased wavefront sensors with the LOOPS v2.0 testbed.," Video Memorie della Societa Astronomica Italiana 1, 5 (Nov. 2019).

[28] Conan, R. and Correia, C., "Object-oriented Matlab adaptive optics toolbox," in [Adaptive Optics Systems IV], Proc. SPIE 9148, 91486C (Aug. 2014). 